\title{
An equivalent additional damping approach to assess vehicle-bridge interaction for train-induced vibration of short-span railway bridges
}

By

\author{
JD YAU
}

Department of Architecture, Tamkang University, New Taipei City, Taiwan 25137

Corresponding author: E-mail: jdyau@mail.tku.edu.tw

Tel: 886-2-26215656\#3139, Fax: 886-2-23959041

MD Martínez-Rodrigo,

Department of Mechanical Engineering and Construction, Universitat Jaume I, 12071 Castellón, Spain

\author{
A Doménech \\ CEL-RAS Argoitectora S.L., 12001 Castellón, Spain
}

\begin{abstract}
Number of Pages: 33
Number of Figures: 16

Number of Tables: 8
\end{abstract}

Jan. 2019

The manuscript has not been previously published, is not currently submitted for review to any other journal, and will not be submitted elsewhere before a decision is made by this journal. 


\title{
An equivalent additional damping approach to assess vehicle-bridge interaction for train-induced vibration of short-span railway bridges
}

\author{
JD Yau ${ }^{1}$, MD Martínez-Rodrigo ${ }^{2}$, A Doménech $^{3}$ \\ ${ }^{1}$ Department of Architecture, Tamkang University, 251 New Taipei City, Taiwan \\ ${ }^{2}$ Department of Mechanical Engineering and Construction, Universitat Jaume I, \\ 12071 Castellón, Spain \\ ${ }^{3}$ CEL-RAS Argoitectora S.L., 12001 Castellón, Spain
}

\begin{abstract}
Constant moving load models are widely adopted in the dynamic analysis of railway bridges under moving trains. However, the use of this simple model may overestimate the resonant response of simply supported bridges if the vehicle-bridge interaction (VBI) effects are neglected, particularly for short spans. To account for the VBI effects, Eurocode 1 allows engineers to consider an additional amount of structural damping which depends on the bridge span. This method is the so-called Additional Damping Method (ADM), and was formulated in order to provide a conservative prediction of the interaction benefit. Nevertheless, the Additional damping method may sometimes yield to an unsafe prediction of the bridge peak response. Considering the interaction benefits, an alternative analytical approach based on an equivalent VBI model under resonant excitations is presented in this investigation. The key parameters dominating the additional damping problem are subsequently identified. According to the numerical demonstrations supplied, the presented approach provides insights that enable an accurate prediction of the additional amount of damping needed in order to account for VBI effects on short simply-supported railway bridges.
\end{abstract}


Keywords: Additional damping method, Eurocode, high-speed railway bridges, moving loads, resonance, vehicle-bridge interaction.

\section{List of acronyms}

$\begin{array}{ll}\text { ADM } & \text { Additional damping method } \\ \text { DoF } & \text { Degrees of freedom } \\ \text { EADA } & \text { Equivalent additional damping approach } \\ \text { ERRI } & \text { European Rail Research Institute } \\ \text { HSR } & \text { High speed rail } \\ \text { MLM } & \text { Moving load model } \\ \text { PC } & \text { Pre-stressed concrete } \\ \text { VBI } & \text { Vehicle-bridge interaction }\end{array}$

\section{Introduction}

The preliminary dynamic analysis of train-induced resonance on railway bridges plays an important role in the design phase of bridge structures since this stage is related to the computer modeling in structural analysis and the building materials used for bridge construction. The time-history analysis of train-bridge systems is a rather time-consuming and laborious task due to the complex coupled differential equations that need to be integrated in the numerical simulation if the different interaction mechanisms that take place are considered. Focusing on the bridge response, moving load models (MLM), which neglect the inertial effects of railway vehicles, are widely adopted for dynamic analysis of railway bridges in most high speed rail (HSR) codes $[1,2]$. However, as the speed of the train approaches a resonant loading condition [3-5], conventional MLM tend to overestimate the bridge response [1-3,6] as the vehicle-bridge interaction (VBI) effects on the train-bridge system are neglected [4-6]. 
To account for VBI benefit, Eurocode 1 [2] provides an additional amount of structural damping that leads to a response reduction under resonance conditions. This approach is the so-called Additional damping method (ADM). The ADM originally proposed by ERRI D214 committee [5] was formulated on the basis of the response of a group of simply supported bridges under the circulation of the ICE-2 and Eurostar trains, in which the trains were modeled as a series of sprung mass units that represent the interaction effect of one bogie travelling over the bridge $[5,8]$. This method is nowadays included in Eurocode 1 [2].

To account for the VBI effects of train-bridge coupling systems on simply supported pre-stressed concrete (PC) bridges, the structural damping ratio $(\zeta)$, the additional damping ratio $(\Delta \zeta)$ proposed in Eurocode 1 [2], and the total structural damping ratio $\left(\zeta_{\text {Total }}\right)$ are respectively prescribed as follows $[1,2]$

$$
\begin{aligned}
& \zeta=\left\{\begin{array}{cl}
1 & L \geq 20 \\
1+0.07(20-L) & L \leq 20
\end{array}\right. \\
& \Delta \zeta=\frac{0.0187 L-0.00064 L^{2}}{1-0.0441 L-0.0044 L^{2}+0.000255 L^{3}} \\
& \zeta_{\text {Total }}=\zeta+\Delta \zeta
\end{aligned}
$$

where $L$ stands for the span length. Then a total structural damping ratio $\left(\zeta_{\text {Total }}\right)$ can be computed incorporating VBI effects in the analysis of short rail bridges using a MLM. The ADM included in Eurocode 1 [2] provides engineers a methodology to quantify the reduction that a railway bridge under resonance experiences due to the energy dissipation associated to the vehicle suspension systems. However, the application of ADM from Eurocode 1 nowadays does not depend on anything else but the bridge length $(L)$ and, specially, does not depend on the vehicle properties. The purpose of the method is to provide a conservative prediction of the reduction that the bridge dynamic response experiences due to the tuning effect of the vehicle masses at resonance. Despite the fact that the ADM should be conservative by definition, some 
case studies prove that the ADM may sometimes overestimate the bridge peak response reduction due to interaction [6], while in other cases the predicted reduction of displacements and accelerations does not reach the values obtained with a detailed representation of the vehicle [7]. Nevertheless, under resonance conditions, moving load models will predict an excessively high response as vehicle-bridge interaction becomes important [8].

A new analytical approach is developed in this investigation in order to provide an accurate prediction of the interaction benefit using a MLM. The proposed method allows identifying the key parameters that dominate the additional damping to be provided in order to adjust the bridge peak response predicted by MLM to that obtained with a detailed representation of the vehicle. According to the numerical verifications supplied, the proposed equivalent additional damping approach (EADA) provides an accurate and safe prediction in order to incorporate the interaction benefit in a constant moving load model. From the numerical demonstrations and parametric studies, it is shown that the proposed equivalent additional damping approach may be a simple and feasible method to account for VBI effects in predicting the response of short bridges under resonance using the MLM.

\section{Problem formulation}

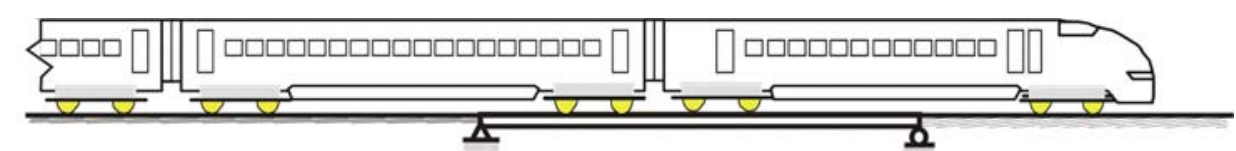

(a)

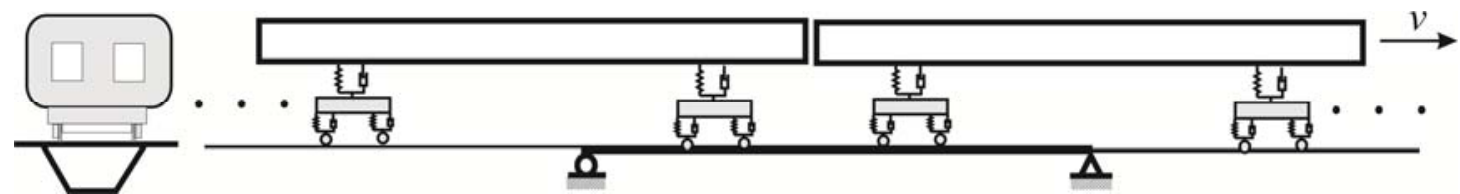

(b)

Fig. 1 Schematic representation of a series of train cars moving over a simple beam (a) conventional HSR train; (b) multi-body vehicle systems 


\subsection{Train-bridge model}

Figure 1 shows a train crossing a single-track simply supported box girder bridge For the analytical formulation of the train-bridge system, the train is modeled as a series of multi-body train cars, as shown in Fig. 1(b) and the bridge as a simply supported beam. Since the moving load models are widely adopted for dynamic analysis of railway bridges in most HSR codes $[1,2]$, the effect of track irregularities on bridge vibrations is disregarded in this study [7-9]. To solve the dynamic interaction problem of the train-bridge system analytically, the following simplifications are admitted:

(1) The bridge is modeled as a linear elastic Timoshenko beam with uniform cross section $[10,11]$

(2) The multi-body train car shown in Fig. 1(b) is represented by the car body, two bogies and four axles, in which the primary and secondary suspension systems are modeled by a set of parallel spring-dashpot systems;

(3) The moving interface between a moving wheel and the beam is regarded as a rigid contact point for conservative evaluation of train-induced bridge response;

(4) The dynamic response of continuous tracks is similar to that of the bridge deck due to the strong constraints of the ballast layer [7-9];

(5) The influence of the ballasted or non-ballasted track has been taken into account only by means of its associated dead mass added to that of the bridge [9]; and

(6) The short bridge needs to be long enough as to allow the rear and front bogies of two adjacent coaches to act on it simultaneously (see Fig. 1). 


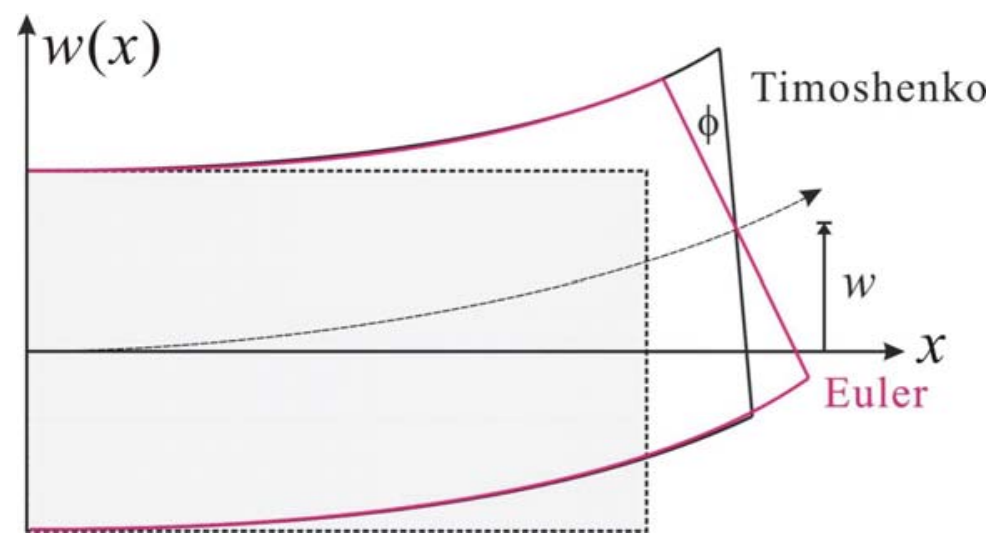

Fig. 2 Timoshenko vs. Bernoulli-Euler beam models

The equations of motion for an elastic Timoshenko beam (see Fig. 2) including shear deformation and rotatory inertia can be expressed as $[10,11]$

$$
\begin{aligned}
& m \frac{\partial^{2} w}{\partial t^{2}}+c_{w} \frac{\partial w}{\partial t}-\kappa A G\left[\frac{\partial^{2} w}{\partial x^{2}}-\frac{\partial \phi}{\partial x}\right]=p(x, t) \\
& \rho I \frac{\partial^{2} \phi}{\partial t^{2}}+c_{\phi} \frac{\partial \phi}{\partial t}-\kappa A G\left[\frac{\partial w}{\partial x}-\phi\right]-E I \frac{\partial^{2} \phi}{\partial x^{2}}=0
\end{aligned}
$$

where the simply supported conditions imply

$$
w(0, t)=w(L, t)=0, E I \phi^{\prime}(0, t)=E I \phi^{\prime}(L, t)=0
$$

In Eqs. (2) and (3) $(\bullet)^{\prime}=\partial(\bullet) / \partial x, \rho=$ mass density of the beam, $\phi=$ angle of rotation of the normal to the mid-surface of the beam, $m=$ mass of the beam per unit length along the $x$-axis, $\left(c_{w}, c_{\phi}\right)=$ deflection and rotation associated damping coefficients, $w(x, t)=$ vertical deflection, $\kappa A G=$ shear rigidity, $\kappa=$ shear coefficient, $E I=$ flexural rigidity, $L=$ length, and $p(x, t)=$ loading function of the train cars moving on the bridge, in which the coach model is composed by one car-body, two bogies, and four wheel-sets (see Fig. 3). 


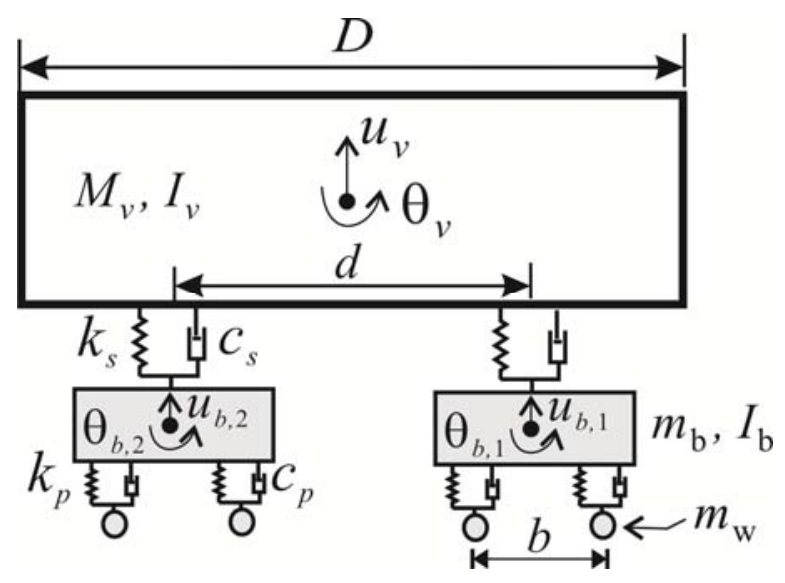

Fig. 3 Multi-body system of a conventional coach model

Considering the regularity of a raw of coaches in a train, the load function $p(x, t)$ shown in Eq. (1) may be written as [9,12]:

$$
p(x, t)=\sum_{n=1}^{N}\left\{\sum_{k=1}^{2}\left[\sum_{i=1}^{2}\left(\frac{-P_{0}}{4}+k_{p} u_{w, k i}(t)+c_{p} \dot{u}_{w, k i}(t)\right) \times \varphi\left(x_{k i, n}, t\right)\right]\right\}
$$

where an over-dot represents derivation with respect to time and the relative vertical displacement $\left(u_{w, k i}\right)$ of the $i$-th wheel-set of the $k$-th bogie with respect to the contact point of the beam is

$$
u_{w, k i}(t)=u_{b, k}-(-1)^{k} b \theta_{b, k} / 2-w\left(x_{k i}, t\right)-r\left(x_{k i}\right) \quad k=1,2
$$

and

$$
\varphi\left(x_{k i, n}, t\right)=\delta\left(x-x_{k i}\right)\left[H\left(t-t_{k i, n}-L / v\right)-H\left(t-t_{k i, n}-L / v\right)\right]_{i=1,2}
$$

Here, $\left(u_{b, k}, \theta_{b, k}\right)$ are, respectively, the vertical displacement and pitching rotation at the midpoint of the $k$-th bogie beneath the train car (see Fig. 3), $N=$ number of train cars, $P_{0}=\left(M_{v}+2 m_{b}+4 m_{w}\right) g=$ total weight of the coach, $M_{v}=$ mass of the car body, $m_{b}$ $=$ mass of the bogie, $m_{w}=$ mass of the wheel-set, $u_{w, k i}=$ vertical displacement of the $i$-th wheelset in the $k$-th bogie, $t_{k i, n}=$ time elapsed until the $i$-th wheelset in the $k$-th bogie of the $n$-th coach reaches the beam, and $r(x)=$ rail roughness. 
Then the matrix equation of motion of the train car with the multi-body system can be written as [12]

$$
\mathbf{M}_{\mathrm{c}} \ddot{\mathbf{u}}_{\mathrm{c}}+\mathbf{C}_{\mathrm{c}} \dot{\mathbf{u}}_{\mathrm{c}}+\mathbf{K}_{\mathrm{c}} \mathbf{u}_{\mathrm{c}}=\mathbf{f}_{\mathrm{c}}
$$

where $\mathbf{M}_{\mathbf{c}}, \mathbf{C}_{\mathbf{c}}$, and $\mathbf{K}_{\mathbf{c}}$ denote the mass, damping, and stiffness matrices, and $\mathbf{u}_{\mathbf{c}}$ and $\mathbf{f}_{\mathbf{c}}$ stand for the displacement and force vectors of the coach model, respectively. All of the system matrices and vectors appearing in Eq. (7) have been given in the Appendix.

\subsection{Solution method}

Let us consider the free vibration of the beam in Eq. (2). Elimination of $\phi$ in Eq.

(2) leads to the following fourth-order differential equation $[10,11]$

$$
m \frac{\partial^{2} w}{\partial t^{2}}+E I \frac{\partial^{4} w}{\partial x^{4}}-\rho I\left[1+\frac{E}{\kappa G}\right] \frac{\partial^{4} w}{\partial t^{2} \partial x^{2}}+\frac{\rho^{2} I}{\kappa G} \frac{\partial^{4} w}{\partial t^{4}}=0
$$

For a simple beam with uniform cross-section, the vibration shape of the displacement may be expressed as [10]

$$
w(x, t)=C_{j} e^{i \omega_{j} t} \sin (j \pi x / L)
$$

where $C_{j}=$ amplitude of the $j$-th vibration mode of the beam and $\omega_{j}=$ the $j$-th circular modal frequency of the beam. Substituting Eq. (9) into Eq. (8) yields the following characteristic equation of the beam free vibration:

$$
\frac{E}{\kappa G}\left(\frac{j r \pi}{L}\right)^{4}\left(\frac{\omega_{j}}{\omega_{E, j}}\right)^{4}-\left[1+\left(1+\frac{E}{\kappa G}\right)\left(\frac{j r \pi}{L}\right)^{2}\right]\left(\frac{\omega_{j}}{\omega_{E, j}}\right)^{2}+1=0
$$

with $r=\sqrt{I / A}=$ radius of gyration and $\omega_{E, j}=(j \pi / L)^{2} \sqrt{E I / m}=$ the $j$-th modal frequency of a simply supported Bernoulli-Euler beam. For a short Timoshenko beam, the frequency ratio of $\left(\omega_{j} / \omega_{E, j}\right)$ always becomes less than unity so that the first term in Eq. (10) may be ignored to yield the natural frequency as [11] 


$$
\omega_{j}=\frac{\omega_{E, j}}{\sqrt{1+\lambda_{j}}} \text { with } \lambda_{j}=\left(\frac{j \pi r}{L}\right)^{2}\left(1+\frac{E}{\kappa G}\right)
$$

From the approximate frequency given by Eq. (11), the fourth derivative with respect to time (fourth term shown in Eq. (8)) is neglected. Under this assumption, Eq. (2) can be rearranged and simplified to the following expression:

$$
\left[m \frac{\partial^{2} w}{\partial t^{2}}-\rho I\left(1+\frac{E}{\kappa G}\right) \frac{\partial^{4} w}{\partial t^{2} \partial x^{2}}\right]+c_{w}\left(\frac{\partial w}{\partial t}-\frac{E r^{2}}{\kappa G} \frac{\partial^{3} w}{\partial t \partial x^{2}}\right)+E I \frac{\partial^{4} w}{\partial x^{4}}=p(x, t)
$$

As indicated in Eqs. (4), (6), and (12), the equations of motion are coupled with the train cars running over the bridge. For a simply supported beam, the dynamic response of the beam can be represented by the summation of sinusoidal functions as [12]

$$
w(x, t)=\sum_{j=1} q_{j}(t) \times \sin \left(\frac{j \pi x}{L}\right)
$$

where $q_{j}(\mathrm{t})=$ generalized coordinate of the $j$-th shape function. Following the Galerkin method [12], the $j$-th generalized equation of motion for the simple beam is expressed as follows:

$$
\begin{aligned}
& \ddot{q}_{j}+2 \zeta_{j} \omega_{j} \dot{q}_{j}+\omega_{j}^{2} q_{j} \\
& =\frac{2 / m L}{\left(1+\lambda_{j}\right)} \sum_{n=1}^{N}\left\{\sum_{k=1}^{2}\left[\sum_{i=1}^{2}\left(\frac{-P_{0}}{4}+k_{p} u_{w, k i}+c_{p} \dot{u}_{w, k i}\right) \psi_{j}\left(\varpi_{j}, t-t_{n, k i}\right)\right]\right\}
\end{aligned}
$$

Here, $\zeta_{j}=$ modal damping, $\varpi_{j}(=j \pi v / L)=$ driving frequency, and

$$
\psi_{j}\left(\varpi_{j}, t-t_{k}\right)=\sin \left(\varpi_{j}\left(t-t_{k}\right)\right) \times\left[H\left(t-t_{k}\right)-H\left(t-t_{k}-L / v\right)\right]
$$

In the right hand side of Eq. (14), if the coupling terms $\left(k_{p} u_{w, k i}+c_{p} \dot{u}_{w, k i}\right)$ are neglected, the equation is reduced to that of a simple beam subject to sequential moving static forces. Let $\lambda_{j}$ be zero in the denominator of Eq. (14), the generalized equation is then reduced to the Bernoulli-Euler beam equation. By solving the generalized equations of the beam in Eq. (14) coupled with the vehicle equations of Eq. (7) using 
conventional direct integration methods [12], the VBI response of the train-bridge system can be computed.

From the viewpoint of flexural strength design, the bending moment is a key magnitude to assess the flexural resistance of a beam. Since the internal moment of a Timoshenko beam is related to the rotation angle $\phi(x, t)$ of the cross section $[9,11]$, the expression of the cross-sectional rotation angle $\phi(x, t)$ is not arbitrary but can be determined from Eq. ( 2 b) by neglecting the inertia term, that is,

$$
-\kappa A G\left[\frac{\partial w}{\partial x}-\phi\right]-E I \frac{\partial^{2} \phi}{\partial x^{2}}=0
$$

Substituting Eq. (13) into Eq. (16) and solving the equation in terms of $\phi(x, t)$ yields

$$
\phi(x, t)=\sum_{j=1} B_{j}(t) \cos \left(\frac{j \pi x}{L}\right) \quad \text { with } \quad B_{j}(t)=\frac{j \pi / L}{1+E /(\kappa G) \times(j \pi r / L)^{2}} q_{j}(t)
$$

Then the dynamic moment $\left(M_{d}\right)$ in the simply supported Timoshenko beam can be represented as [9]

$$
M_{d}(x, t)=-E I \frac{d \phi}{d x}=\sum_{j=1}\left[\frac{E I(j \pi / L)^{2}}{1+E /(\kappa G) \times(j \pi r / L)^{2}} q_{j}(t) \times \sin \left(\frac{j \pi x}{L}\right)\right]
$$

By letting $\kappa G \rightarrow \infty$ in the denominator in Eq. (18), one can obtain the dynamic bending moment of a simply supported Bernoulli-Euler beam. In this study, the dynamic response in terms of the bending moment will be used to evaluate the dynamic amplification phenomenon for a simple beam subject to moving train loads.

\section{Equivalent additional damping approach}

To account for VBI effects, ERRI D-214 and Eurocode $1[1,2,5,6]$ proposed an ADM to reduce the bridge response when performing dynamic analyses of bridges using the moving load models $[1,2]$. However, the ADM may lead to an excessive response reduction for short bridges under resonance. As can be seen from Eq. 1(b), the damping value assumed for the structure applying the ADM is increased as a percentage of the span length. As stated in the introduction, the method was 
developed by the ERRI-D214 [5] considering the ICE-2 and the Eurostar trains in the calibration process. The trains were schematized as a sequence of sprung mass units [8]. As for the bridges, a span range from 5 to $30 \mathrm{~m}$ was covered, considering a constant linear mass for each span, three damping ratios $(0.5 \%, 1 \%$ and $2 \%)$ and different values of the flexural stiffness. The additional damping was obtained as the safe or lower bound estimate for each of the spans, and for this reason the ADM is conservative in the majority of the situations, underestimating the interaction benefit. On the other hand, due to the wide range of possible values that can take the mechanical parameters which define the train-bridge system, some case studies can be found in which the ADM leads to a non-conservative prediction of the bridge response [8].

To overcome the drawbacks indicated above, this study presents an alternative approach to formulate the additional structural damping, in which the key parameters that govern the additional damping needed for each scenario are detected by means of an analytical formulation.

\subsection{Modelling of the equivalent additional damping}

To simulate the VBI effect for a train traveling over a bridge in numerical computation, according to ERRI-D214 [5], a coach is decomposed into two equivalent identical single-DoF oscillators, each of which represents the vertical fundamental modal system of a half coach (see Fig. 4). With is consideration, the train is modeled as a series of sprung mass units moving on a bridge in the following theoretical formulation. 


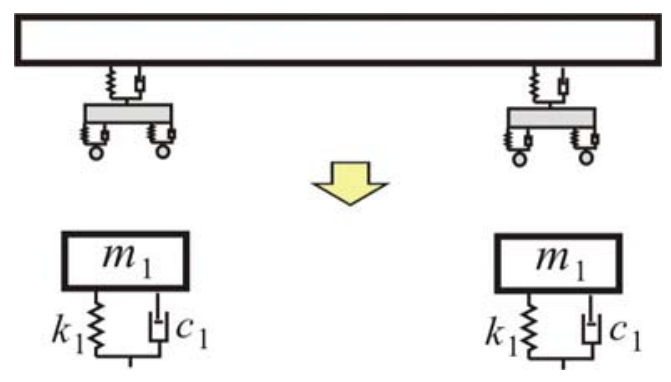

Fig. 4 Simplified model of equivalent sprung mass units of a conventional coach

Let us consider the simplified oscillators in a coach shown in Fig. 4. By performing an eigenvalue analysis, the first vertical modal properties of the planar multi-body coach are obtained. The modal mass and stiffness of the half coach can be used to represent the structural properties of the equivalent single-DoF oscillator as follows:

$$
m_{1}=\frac{1}{2} \varphi_{1}^{\mathrm{T}} \mathbf{M}_{\mathbf{c}} \boldsymbol{\varphi}_{1}, \quad k_{1}=\frac{1}{2} \boldsymbol{\varphi}_{1}^{\mathrm{T}} \mathbf{K}_{\mathrm{c}} \boldsymbol{\varphi}_{1}
$$

Here, $\varphi_{1}$ stands for the first vertical modal shape of the $2 \mathrm{D}$ vehicle model. Let us denote the damping of the equivalent system as $c_{1}$, the damping ratio of the equivalent suspension system can then be expressed as

$$
\xi_{v 1}=c_{1} / 2 m_{1} \omega_{v 1}, \quad \text { with } \omega_{v 1}=\sqrt{k_{1} / m_{1}}
$$

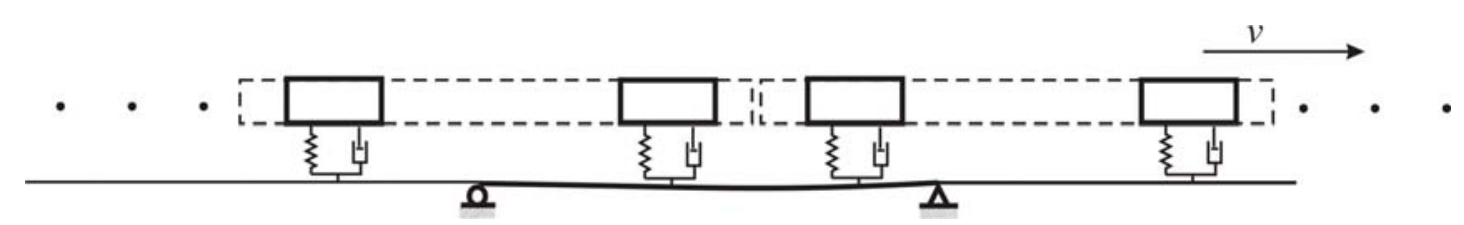

Fig. 5 Tuning effects of two running coaches on a short bridge

When the front and rear equivalent single-DoF oscillators of two adjacent coaches are simultaneously traveling over the bridge, as represented in Fig. 5, the inertial effects of the masses and the energy dissipation through the suspension systems offer a tuning action originating a reduction of the bridge vibration levels [18]. 
For a simply supported beam, its dynamic displacement is mainly governed by its fundamental mode [7]. For this reason, let us consider Eqs. (4) and (5) that the beam displacement $w(x, t)$ affecting the train loadings acting on the beam can be represented by the first mode of the simple beam. Then a modal system of equations can be obtained by combining the front and rear equivalent single-DoF oscillators of two adjacent coaches with the first vibration mode of the bridge (only this mode is considered to represent the dynamic behavior of the bridge in the analytical formulation). The resulting model is represented in Fig 6(a), where the structural properties of $\left(M_{1}, C_{1}, K_{1}\right)$ stand for the first modal properties of the mass, damping, stiffness of the bridge, respectively. A harmonic force $F_{0} e^{i \omega t}$ is used to simulate the periodic excitation of a regular set of train loads acting on the bridge.

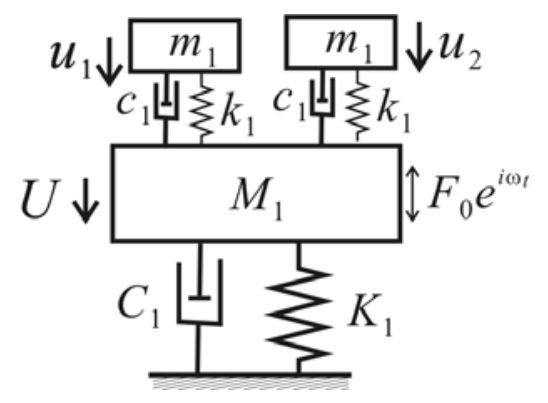

(a)

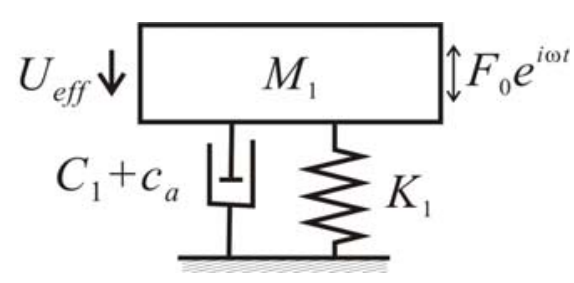

(b)

Fig. 6 Mathematical models for the coach-bridge coupling system;

(a) simplified model with two tuning units; (b) equivalent model including additional damping $C a$.

The equation of motion of the model represented in Fig 6(a) can be expressed in a matrix form as 


$$
\begin{aligned}
{\left[\begin{array}{ccc}
M_{1} & & \\
& m_{1} & \\
& & m_{1}
\end{array}\right]\left\{\begin{array}{l}
\ddot{U} \\
\ddot{u}_{1} \\
\ddot{u}_{2}
\end{array}\right\} } & {\left[\begin{array}{ccc}
C_{1}+2 c_{1} & -c_{1} & -c_{1} \\
-c_{1} & c_{1} & 0 \\
-c_{1} & 0 & c_{1}
\end{array}\right]\left\{\begin{array}{l}
\dot{U} \\
\dot{u}_{1} \\
\dot{u}_{2}
\end{array}\right\} } \\
& +\left[\begin{array}{ccc}
K_{1}+2 k_{1} & -k_{1} & -k_{1} \\
-k_{1} & k_{1} & 0 \\
-k_{1} & 0 & k_{1}
\end{array}\right]\left\{\begin{array}{c}
U \\
u_{1} \\
u_{2}
\end{array}\right\}=\left\{\begin{array}{c}
F_{0} e^{i \omega t} \\
0 \\
0
\end{array}\right\}
\end{aligned}
$$

For the first resonance of the system, that is, $\omega=\sqrt{K_{1} / M_{1}}$, the response amplitude $\bar{U}$ of the main mass $\left(M_{1}\right)$ in steady state becomes

$$
\bar{U}=\frac{F_{0}}{K_{1}} \times\left[2 \zeta i+\frac{2 \mu_{1} r_{1}\left(r_{1}+2 \xi_{v 1} i\right)}{\left(1-r_{1}^{2}\right)-2 \xi_{v 1} r_{1} i}\right]^{-1}
$$

where $\zeta=C_{1} /\left(2 M_{1} \omega\right), \quad \mu_{1}=m_{1} / M_{1}=$ modal mass ratio, and $r_{1}=\omega_{v 1} / \Omega=$ frequency ratio of the coach-bridge system. As can be deduced after Eq. (22), the resonant response amplitude $(\bar{U})$ of the main mass is dominated by the three key parameters $\left(\mu_{1}, \xi_{v 1}, r_{1}\right)$. In what follows, a simplified formula to calculate the additional structural damping ratio in terms of these non-dimensional parameters for MLM to account for VBI effect will be unveiled.

\subsection{Equivalent Additional Damping Model}

Train-induced resonance of the bridge at primary resonant speeds $[7,8,13]$ is the main concern in this study. Let us consider the simplified MDOF system shown in Fig. 6a for the VBI model. Once the main mass of the MDOF system is in resonance, the equivalent damping $\left(C_{1}+c_{a}\right)$ of the SDOF system (see Fig. $6 \mathrm{~b}$ ) can be adjusted to provide the same resonant amplitude of the main mass (see Fig. 6a). To incorporate the VBI effects in the MLM based on the above description, an additional damping $C a$ is added to the structural damping constant $C_{1}$ in the main system, that is, $C_{1}+C_{a}$ 
(see Fig. 6(b)). Then the governing equation in the frequency domain of the new equivalent system becomes:

$$
\left[\left(K_{1}-M_{1} \omega^{2}\right)+i\left(C_{1}+C_{a}\right) \omega\right] \bar{U}_{e f f}=F_{0}
$$

Let us introduce the resonant condition of $\omega=\Omega=\sqrt{K_{1} / M_{1}}$ into Eq. (23). The response amplitude of the equivalent system including the additional damping becomes

$$
\bar{U}_{\text {eff }}=\frac{F_{0}}{i\left(C_{1}+c_{a}\right) \Omega}=\frac{F_{0} / K_{1}}{2\left(\zeta+\Delta \xi_{a}\right) i}
$$

where $\Delta \xi_{a}=$ equivalent additional damping ratio of the bridge. If the amplitude $|\bar{U}|$ in Eq. (22) is equated to $\left|\bar{U}_{\text {eff }}\right|$ in Eq. (24), the following expression for the additional damping ratio $\Delta \xi_{a}$ can be depicted

$$
\Delta \xi_{a}=\mu_{1} r_{1}\left|\frac{r_{1}+2 \xi_{v 1} i}{\left(1-r_{1}^{2}\right)-2 \xi_{v 1} r_{1} i}\right|
$$

As indicated in Eq. (25), three key parameters: (1) the mass ratio, $\mu_{1}$, (2) the frequency ratio, $r_{1}$, and (3) the effective suspension damping ratio, $\xi_{v 1}$, are identified to govern VBI effects in short bridges. The principal advantage of the proposed EADA method when corresponding to the ADM from Eurocode 1 is that, preserving a clear simplicity, it takes into account the main ratios of train-bridge system, while the ADM only depends on the bridge length. This is referred to as equivalent additional damping approach (EADA) in this study. Moreover, if the mass ratio $\mu_{1}$ is fixed, Eq. (25) shows that stiff springs and heavy damping in the coach suspension system may lead to higher additional damping. By contrast, let the frequency ratio $r_{1}$ become zero, the additional damping ratio in Eq. (25) vanishes and the VBI problems reduces to a constant moving load problem. 
For a practical train car, the vertical frequency ratio $r_{1}$ of a coach to the first bending frequency of a short bridge is considerably less than unity based on the strict requirements of riding comfort for high speed trains. By this approximation of $\left|\left(1-r_{1}^{2}\right)-2 \xi_{v 1} r_{1}\right| \square 1$ and following Eq. (25), a simplified additional damping ratio in may be obtained as

$$
\Delta \xi_{e} \approx \mu_{1} r_{1} \sqrt{r_{1}^{2}+\left(2 \xi_{v 1}\right)^{2}}
$$

Obviously, Eq. (26) gives a simple form for railway bridge engineers to predict the additional damping ratio of a short bridge. Moreover, it provides physical insights into the ADM to determine a suitable amount of additional damping for the short bridge that can account for the VBI effects in MLM.

From the viewpoint of vibration control, the mass ratio $\left(\mu_{1}\right)$ can be regarded as a tuning unit in reducing the vibration of the main mass while the frequency and vehicle damping ratio $\left(r_{1}, \xi_{v 1}\right)$ offer a vibration regulator to adjust the interaction between the main mass $\left(M_{1}\right)$ and the tuning masses $\left(m_{1}\right)$. The application of the equivalent additional damping to VBI effect on bridge vibrations will be conducted in the following parametric study.

Table 1 Coach system parameters for ICE2 [8]

\begin{tabular}{cccccccc}
\hline$M_{v}(\mathrm{t})$ & $m_{b}(\mathrm{t})$ & $m_{w}(\mathrm{t})$ & $I_{v}\left(\mathrm{t} \cdot \mathrm{m}^{4}\right)$ & $I_{b}\left(\mathrm{t} \cdot \mathrm{m}^{4}\right)$ & $D(\mathrm{~m})$ & $d(\mathrm{~m})$ & $b(\mathrm{~m})$ \\
\hline 33.93 & 2.37 & 1.73 & 1971 & 1.24 & 26.4 & 17.94 & 2.5 \\
\hline$k_{s}(\mathrm{kN} / \mathrm{m})$ & $c_{s}(\mathrm{kN} \cdot \mathrm{s} / \mathrm{m})$ & $k_{p}(\mathrm{kN} / \mathrm{m})$ & $c_{p}(\mathrm{kN} \cdot \mathrm{s} / \mathrm{m})$ & $\xi_{v 1}(\%)$ & $f_{v 1}(\mathrm{~Hz})$ & $f_{p 1}(\mathrm{~Hz})$ & $m_{1}(\mathrm{t})$ \\
\hline 301 & 6 & 1598 & 20 & 3.82 & 0.64 & 0.75 & 17.24 \\
\hline
\end{tabular}

Table 2 Coach system parameters for ETR500Y $[8,18]$

\begin{tabular}{cccccccc}
\hline$M_{v}(\mathrm{t})$ & $m_{b}(\mathrm{t})$ & $m_{w}(\mathrm{t})$ & $I_{v}\left(\mathrm{t} \cdot \mathrm{m}^{4}\right)$ & $I_{b}\left(\mathrm{t} \cdot \mathrm{m}^{4}\right)$ & $D(\mathrm{~m})$ & $d(\mathrm{~m})$ & $b(\mathrm{~m})$ \\
\hline 34.23 & 2.76 & 1.583 & 1624 & 2.5 & 26.1 & 17.75 & 3 \\
\hline$k_{s}(\mathrm{kN} / \mathrm{m})$ & $c_{s}(\mathrm{kN} \cdot \mathrm{s} / \mathrm{m})$ & $k_{p}(\mathrm{kN} / \mathrm{m})$ & $c_{p}(\mathrm{kN} \cdot \mathrm{s} / \mathrm{m})$ & $\xi_{v 1}(\%)$ & $f_{v 1}(\mathrm{~Hz})$ & $f_{p 1}(\mathrm{~Hz})$ & $m_{1}(\mathrm{t})$ \\
\hline 182.7 & 16.35 & 807.5 & 7.5 & 7.38 & 0.49 & 0.63 & 17.50 \\
\hline
\end{tabular}


Table 3 Properties of the bridge models. [8]

\begin{tabular}{ccccccc}
\hline Type & $L(\mathrm{~m})$ & $m(\mathrm{t} / \mathrm{m})$ & $E I\left(\mathrm{kN}-\mathrm{m}^{4}\right)$ & $f_{1}(\mathrm{~Hz})$ & $\operatorname{vr}(\mathrm{km} / \mathrm{h})$ & $\mathrm{M}_{1}(\mathrm{t})$ \\
\hline S-15.84 & 15.84 & 20.97 & $4.11 \times 10^{7}$ & 8.76 & 833 & 166.1 \\
S-15.66 & 15.66 & 19.91 & $3.48 \times 10^{7}$ & 8.47 & 796 & 155.9 \\
\hline
\end{tabular}

Table 4 Prediction of additional damping ratios of the bridge models [8]

\begin{tabular}{cccccc}
\hline Type & $\begin{array}{c}\mu_{1} \\
\left(\mathrm{~m}_{1} / \mathrm{M}_{1}\right)\end{array}$ & $\begin{array}{c}r_{1} \\
(\%)\end{array}$ & $\begin{array}{c}\xi_{\mathrm{v} 1} \\
(\%)\end{array}$ & $\begin{array}{c}\Delta \xi_{a} * \\
(\%)\end{array}$ & $\begin{array}{c}\Delta \xi_{e} * * \\
(\%)\end{array}$ \\
\hline ICE-2/S-15.84 & 0.104 & 7.31 & 3.76 & 0.08 & 0.08 \\
ETR500Y/S-15.66 & 0.112 & 5.81 & 7.20 & 0.10 & 0.10 \\
\hline
\end{tabular}

*Eq. (25) **Eq. (26)

Table 5 Structual damping and additional damping ratios of the bridge models.

\begin{tabular}{cccccc}
\hline Type & $\zeta(\%)$ & $\Delta \zeta *(\%)$ & $\zeta_{\text {Total }}=\zeta+\Delta \zeta(\%)$ & $\Delta \xi_{e}(\%)$ & $\xi_{\text {Total }}=\zeta+\Delta \xi_{e}(\%)$ \\
\hline S-15.84 & 1.29 & 0.65 & 1.94 & 0.08 & 1.37 \\
S-15.66 & 1.30 & 0.65 & 1.95 & 0.10 & 1.40 \\
\hline * Eq (1b) & & &
\end{tabular}

* Eq. (1b)

\section{Numerical verifications}

In order to demonstrate the capability and reliability of the equivalent additional damping ratio derived in Eq. (25) to account for the VBI effects in the MLM for short bridges, the response curves of maximum acceleration $\left(a_{\max }\right)$ of the bridge $v s$. speed $(v)$ are computed and compared with the corresponding results presented in Ref. [6]. Such a plot between $a_{\max }$ and $v$ is called " $a_{\max }-v$ plot" in this study. The system properties of ICE-2 and ETR500Y trains and the short bridges evaluated are listed in Tables 1-3, respectively. Here, $v_{r}\left(=D f_{1}\right)=$ the primary resonant speed of the bridge [13], $f_{1}=$ bridge fundamental frequency, and $\left(f_{v 1}, f_{p 1}\right)$ are the vertical and pitching frequencies of the planar coach [12]. Table 4 lists the equivalent additional damping ratios of $\left(\Delta \xi_{a}, \Delta \xi_{e}\right)$ obtained from Eqs. (24) and (25) for the bridges S-15.84 and S-15.66 with respect to the ICE-2 and the ETR500Y trains, respectively $[8,18]$. As shown in Table 
4, the identical values of the additional damping ratios of $\left(\Delta \xi_{a}, \Delta \xi_{e}\right)$ demonstrate that the presented simplified formula in Eq. (26) can be used to approximate the detailed formula in Eq. (25). Moreover, Table 5 lists the total structural damping $\left(\zeta_{\text {Total }}, \xi_{\text {Total }}\right)$ of the bridges computed by the ADM proposed by the Eurocode 1 and the EADA presented in Section 3, respectively.

For the purpose of comparisons, the Bernoulli-Euler beam theory [8] is adopted for the beams listed in Table 3. The Newmark method with constant average acceleration, i.e., with $\beta=0.25$ and $\gamma=0.5$ for its unconditional stability [19] is employed to solve the VBI problem, in which a small time step $(0.0002 \mathrm{~s})$ is used for computational accuracy. The ending time is $t_{e n d}=(L+N D) / v$. Here, $N=$ total number of coaches constituting the train $(N=10)$.

Following the dynamic analysis of the MLM and VBI models based on the Bernoulli-Euler beam reduced from the Timoshenko beam theory formulated in Section 2, Figs. 7(a) and 7(b) show the $a_{\max }-v$ plot for the short bridges under the action of ICE-2 and ETR500Y trains, respectively in a velocity range swept from 200 to $450 \mathrm{~km} / \mathrm{h}$ with an interval of $2.5 \mathrm{~km} / \mathrm{h}$. As indicated, the present resonant peaks at the sub-resonant speeds of $\left(v_{r} / 2, v_{r} / 3\right)[14,15]$ are in agreement with those obtained from Domenech et al. [8]. Obviously, the numerical comparisons show that the proposed equivalent additional damping approach (EADA) can give consistent predictions of the order of VBI effects to be incorporated in the computations with MLM in order to obtain the response of the short bridges under resonance conditions. For illustration, the corresponding time history responses of midpoint displacement of the bridges subject to ICE2 and ETR500Y have been plotted in Figs. 8(a) and 8(b), respectively. The results obtained using the proposed method (MLM+EADA) are in agreement with those considering VBI effects. But the train-induced bridge response 
computed by the MLM+ADM is significantly underestimated.

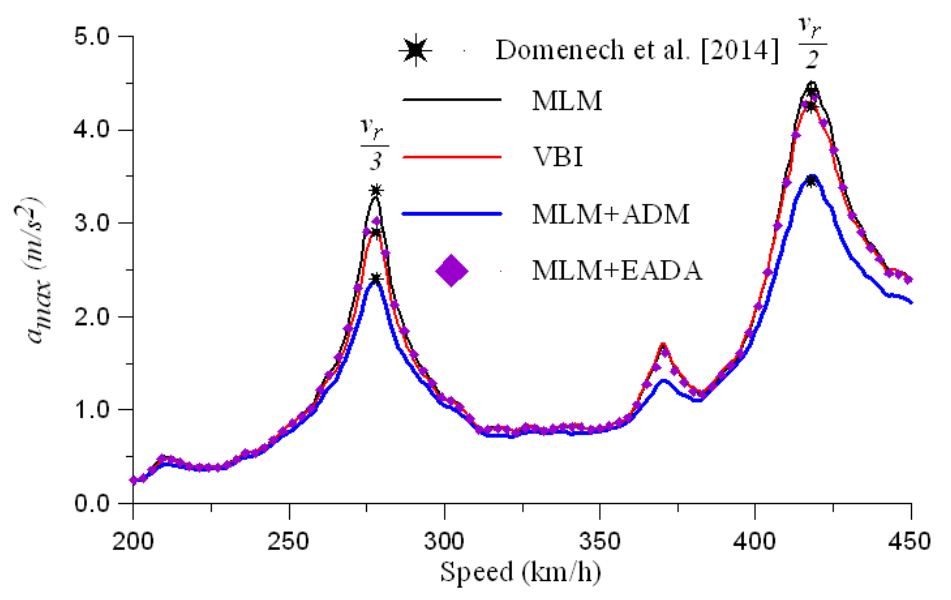

(a)

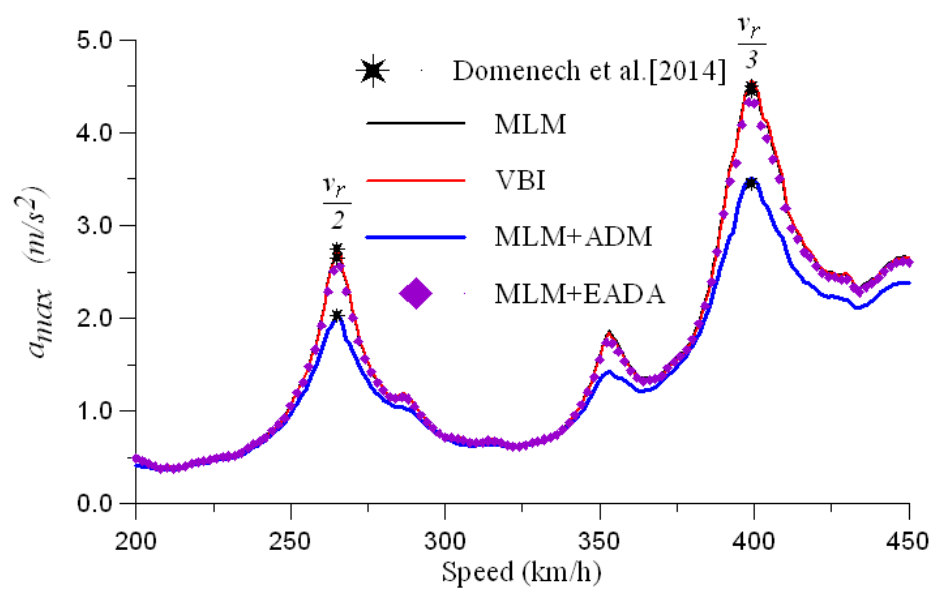

(b)

Fig. 7 Comparison of $a_{\max }-v$ plot for the short bridges:

(a) the short bridge (S-15.84) subject to ICE2; (b) the short bridge (S-15.66) subject to ETR500Y 


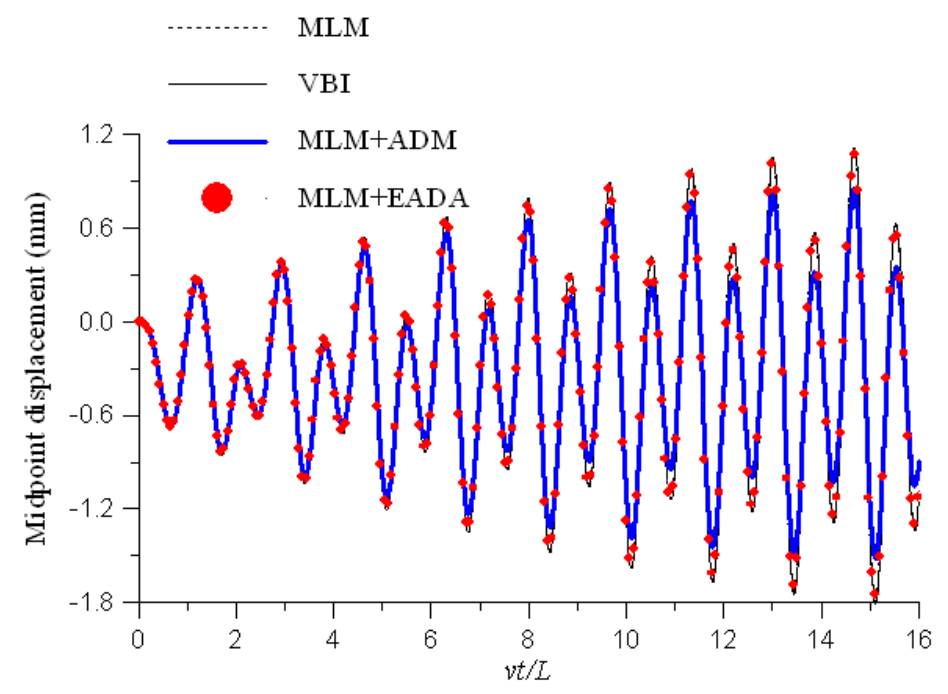

(a)

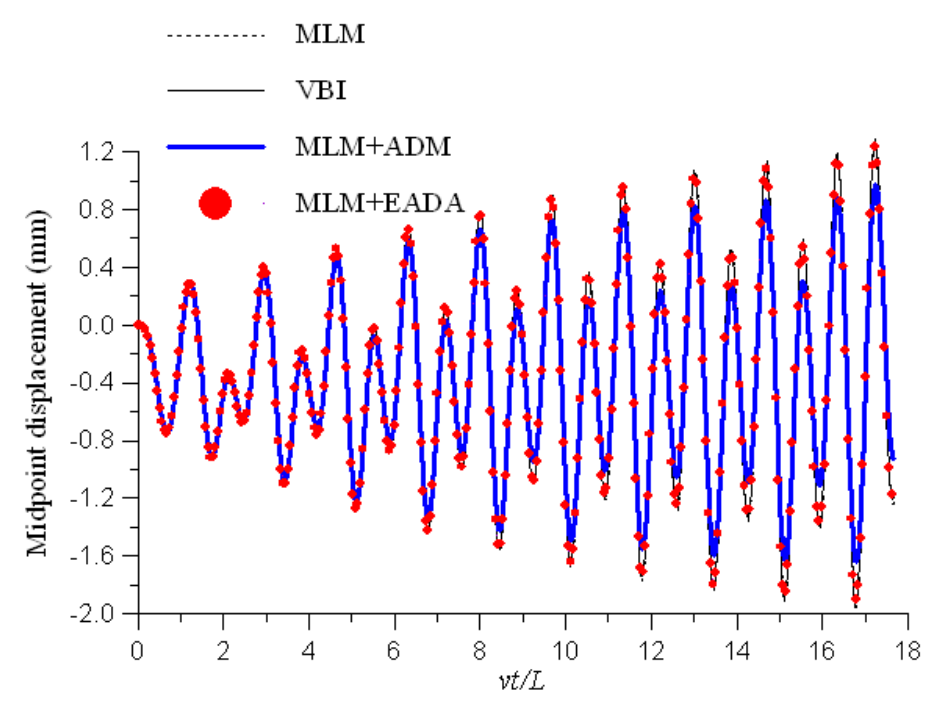

(b)

Fig. 8 Comparison of time history response of midpoint displacement of the bridges

(a) the short bridge (S-15.84) subject to ICE2; (b) the short bridge (S-15.66) subject to ETR500Y

Let us now observe the $a_{\max }-v$ plot shown in Fig. 7(a). The figure shows that the sub-resonant peaks of the bridge S-15.66 subject to the ETR500Y train are almost identical for both of the MLM and the VBI models. In this case, the interaction benefit vanishes almost completely due to the low suspension stiffness of the ETR500Y coach, as listed in Table 2. As the fundamental frequency of the short 
bridge is much higher than the frequency of the vehicle, a low tuning effect of the vehicle masses on the bridge vibration is induced on the vehicles with lower suspension stiffness. In the following section, the parametric study of VBI effects on the bridge response will be conducted using the proposed equivalent additional structural damping for the bridges shorter than $15 \mathrm{~m}$.

Table 6 Properties of the Timoshkeno beam models.

\begin{tabular}{|c|c|c|c|c|c|c|c|}
\hline Type & $\begin{array}{c}L \\
(\mathrm{~m})\end{array}$ & $\begin{array}{c}m \\
(\mathrm{t} / \mathrm{m})\end{array}$ & $\begin{array}{c}E I \\
\left(\mathrm{kN}-\mathrm{m}^{2}\right)\end{array}$ & $\begin{array}{l}\kappa A G \\
(\mathrm{kN})\end{array}$ & $\begin{array}{c}f_{1} \\
(\mathrm{~Hz})\end{array}$ & $\begin{array}{l}M_{1} \\
(\mathrm{t})\end{array}$ & $\begin{array}{c}v_{r}(\mathrm{~km} / \mathrm{h}) \\
\text { ICE-2 / ETR500Y }\end{array}$ \\
\hline S-12 & 12 & 17.5 & $1.98 \times 10^{7}$ & $2.30 \times 10^{7}$ & 11.25 & 105 & $1069 / 1057$ \\
\hline S-14 & 14 & 19.5 & $3.04 \times 10^{7}$ & $2.65 \times 10^{7}$ & 9.70 & 137 & $921 / 911$ \\
\hline
\end{tabular}

Table 7 Parameters of EADA for ICE-2 and ETR500Y

\begin{tabular}{ccccc}
\hline \multicolumn{3}{c}{$\mu_{1}\left(\mathrm{~m}_{1} / \mathrm{M}_{1}\right)$} & \multicolumn{2}{c}{$r_{1}(\%)$} \\
\cline { 1 - 2 } Type & ICE-2 & ETR500Y & ICE-2 & ETR500Y \\
\cline { 1 - 3 } S-12 & 0.162 & 0.163 & 5.69 & 4.36 \\
S-14 & 0.127 & 0.125 & 6.60 & 5.05 \\
\hline
\end{tabular}

Table 8 Structual damping and additional damping ratios

\begin{tabular}{ccccll}
\hline Type & $\zeta(\%)$ & $\Delta \zeta^{*}(\%)$ & $\zeta_{\text {Total }}=\zeta+\Delta \zeta(\%)$ & $\Delta \xi_{e}{ }^{* *}(\%)$ & $\xi_{\text {Total }}=\zeta+\Delta \xi_{e}(\%)$ \\
\hline S-12 & 1.56 & 0.48 & 2.04 & $0.09(\mathrm{ICE}-2)$ & 1.65 (ICE-2) \\
& & & & $0.11(\mathrm{ETR})$ & 1.67 (ETR) \\
S-14 & \multirow{2}{*}{1.42} & 0.62 & 2.04 & $0.08(\mathrm{ICE}-2)$ & $1.50(\mathrm{ICE}-2)$ \\
& & & & $0.10(\mathrm{ETR})$ & $1.52(\mathrm{ETR})$ \\
\hline
\end{tabular}

*Eq. (1b) proposed by EN 1991-2, **Eq. (26)

\section{Parametric studies}

Considering the effects of shear deformation and rotatory inertia for short bridges, the Timoshenko beam formulated in Section 2.1 is employed in this section to investigate the dynamic behaviour of short-span bridges in the following examples. For the sake of comparison in the parametric studies, four moving load models (MLM, VBI, MLM+ADM, MLM+EADA) and two short bridges (S-12, S-14) are considered, 
respectively. The properties of the two short bridges S-12 and S-14 are listed in Table 6. The presented data correspond to empirical values of PC bridges included in references $[16,17]$. Let us consider the same ICE-2 and ETR500Y train models given in the previous section. The corresponding primary resonant speeds $\left(v_{r}\right)$ for the bridges S-12 and S-14 are listed in Table 6. As indicated, the primary resonant speeds are much higher than the operation speeds in current HSR systems. However sub-resonance situations will be induced on the railway bridge as well if the train speed coincides with any of the sub-resonant conditions, i.e., $v_{r} /\left.n\right|_{n=2,3,4 . .}$. Here, $n$ represents the number of complete oscillation cycles experienced by the beam during the passage of two adjacent loads $[14,15]$. Thus, the velocity-range swept from 100 to $420 \mathrm{~km} / \mathrm{h}$ at intervals of $2.5 \mathrm{~km} / \mathrm{h}$ will be considered in the following examples.

In order to compute the equivalent additional damping ratio $\Delta \xi_{e}$ in Eq. (26) for the short bridges $(\mathbf{S}-12, \mathbf{S}-14)$, the parameters $\left(\mu_{1}, r_{1}\right)$ required for computing the equivalent additional damping ratios have been listed in Table 7 . Based on the additional damping ratios shown in Eqs. (1b) and (26), the total structural damping $\left(\zeta_{\text {Total }}, \xi_{\text {Total }}\right)$ of the bridges $(\mathbf{S - 1 2}, \mathbf{S}-14)$ calculated by the ADM and the herein proposed EADA for the ICE-2 and the ETR500Y trains are listed in Table 8. In the following examples, the maximum acceleration and bending moment in the beams due to the passage of the high speed trains are presented.

\subsection{VBI effects on the maximum acceleration of the beams}

Since the maximum vertical acceleration on a railway bridge deck can lead to premature ballast destabilization of the track system, the acceleration response of the bridge deck can be regarded as an indicator of train traffic safety and it is considered in this example. Figures 9-12 show the $a_{\max }-v$ plot of the two short bridges subject to 
the ICE-2 and ETR500Y trains. As indicated, the proposed MLM+EADA method provides a consistent prediction for MLM to account for VBI effect on the bridge response under resonance conditions. In the aforementioned figures, the symbol "\#” denotes that the second bending mode of the bridge was excited by the train loads [14], and the symbol “*” represents sub-resonant speeds which coincide with cancellation conditions, and therefore are not detectable [20,21]. From the numerical comparisons shown in Section 4 [16], it can be detected that the ADM [2] leads to a considerable response reduction at sub-resonant speeds in the $a_{\max }-v$ plots (see Figs. 9-12) due to overestimation of additional damping (see Table 8). Compared to the $a_{\max }-v$ plots in Figs. 7 and 8 , the distribution of sub-resonant peaks in the $a_{\max }-v$ plots becomes denser. This is related to the higher value of the fundamental frequency of the bridges shorter than $15 \mathrm{~m}$ under consideration. This fact indicates that a short HSR bridge will experience multiple sub-resonances due to the train passage in operation speeds. This point should be incorporated in HSR design phase to evaluate the impact effect of a train moving on a short bridge.

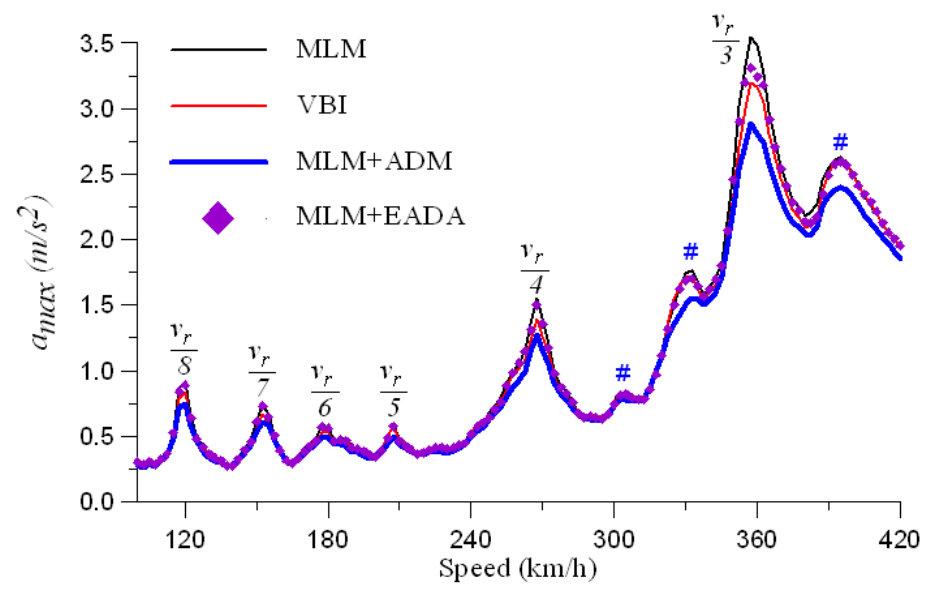

Fig. $9 a_{\max }-v$ plot for the short bridge (S-12) subject to ICE2 


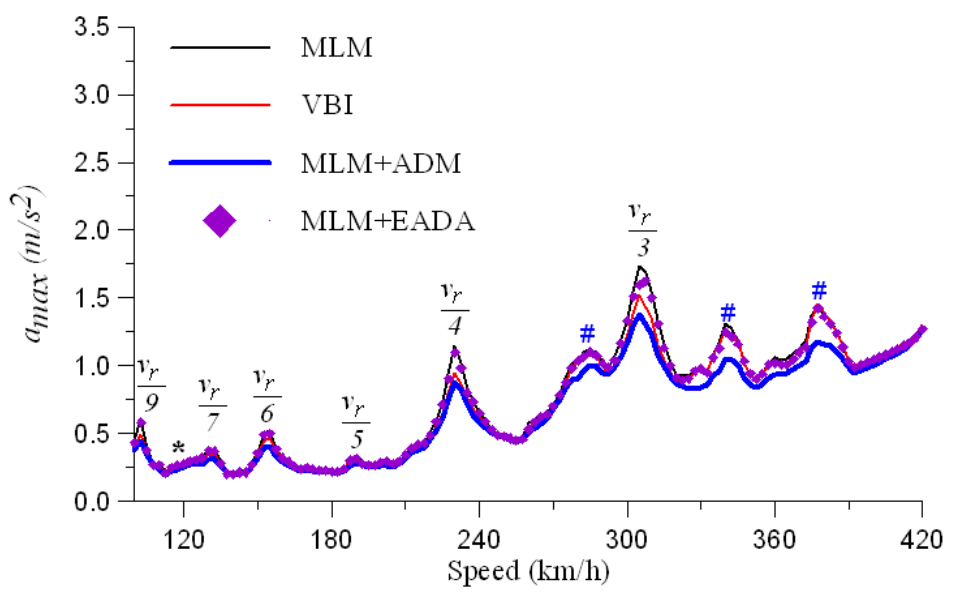

Fig. $10 a_{\max }-v$ plot for the short bridge (S-14) subject to ICE2

As described in Section 4, the interaction benefit in the case study of ETR500Y vanishes almost completely due to the low suspension stiffness of its coaches. The same dynamic phenomena may be also observed in Figs. 11 and 12, respectively. Thus for the coaches with lower suspension stiffness, the ADM will overestimate the VBI benefits in the case of short bridges. In the following section, a parametric analysis is conducted focusing on the dynamic internal bending moment amplification in the structures.

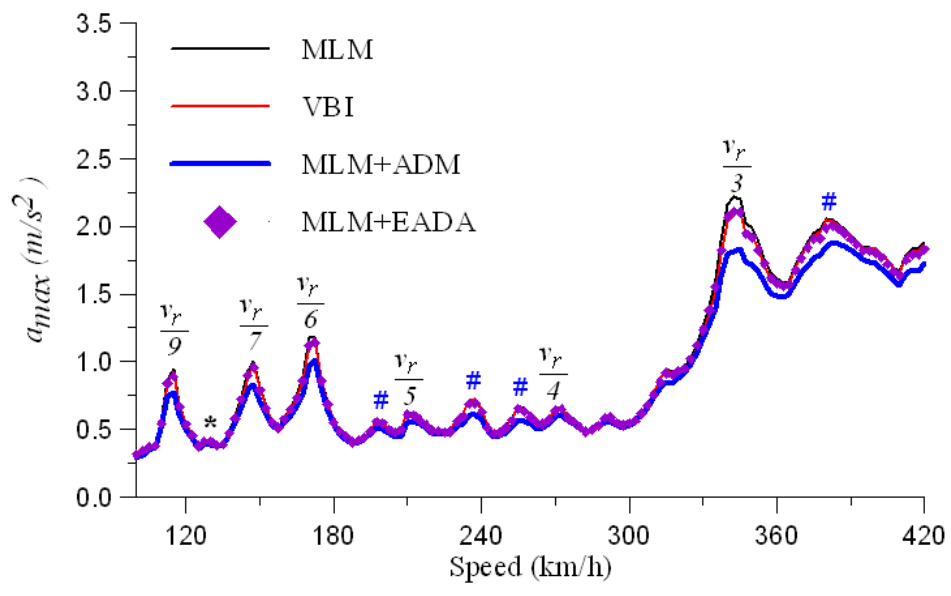

Fig. $11 a_{\max }-v$ plot for the short bridge (S-12) subject to ETR500Y 


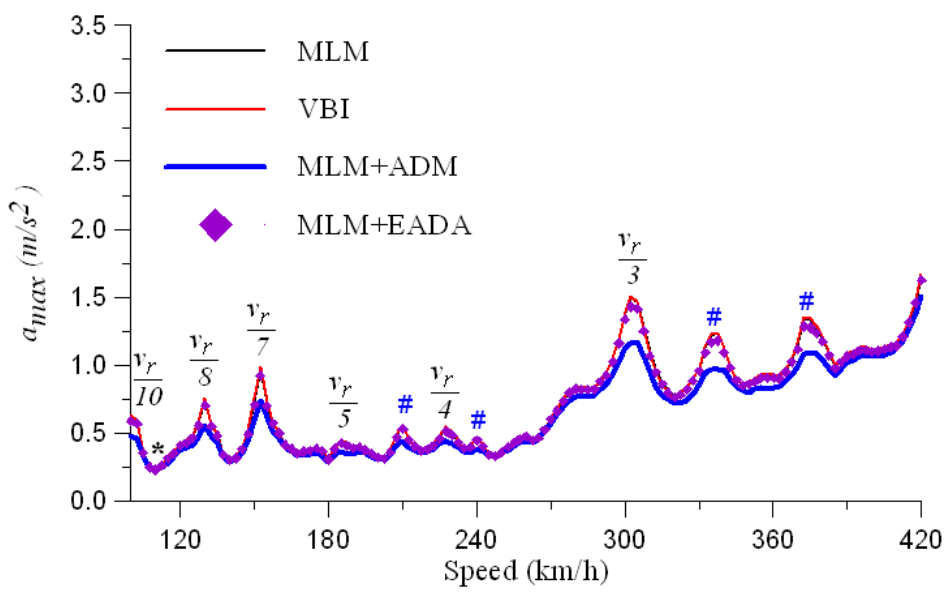

Fig. $12 a_{\max }-v$ plot for the short bridge (S-14) subject to ETR500Y

\subsection{VBI effect on dynamic amplification of bending moment in the beams}

In bridge design practice, the dynamic amplification factor $(D A F)$ is usually used to account for the dynamic effect on the bridge response resulting from the passage of moving vehicles. In this study, the DAF of the bending moment in a simple beam is denoted as $D A F_{M}$ and defined as [22]:

$$
D A F_{M}=\frac{\left|M_{d}(L / 2, t)\right|_{\text {max }}}{M_{s}(L / 2)_{\max }}
$$

where $M_{d}(L / 2, t)$ stands for the dynamic bending moment at mid-span of the beam, as shown in Eq. (18), and $\left|M_{d}(L / 2, t)\right|_{\max }$ and $M_{s}(L / 2)_{\max }$ denote the maximum dynamic and static bending moment at mid-span of the beam caused by the moving train loads, respectively.

Figures 13-16 show the response curves of the $D A F_{M}$ against the train speed $(v)$ for the ICE2 and the ETR500Y trains moving on the S-12 and S-14 bridges, respectively. Here, the relation between $D A F_{M}$ and $v$ is called " $D A F_{M}-v$ plot". As expected, the proposed MLM+EADA can provide consistent predictions for MLM to account for VBI effect on the $D A F_{M-V}$ plots in resonance conditions. The ADM [2] still underestimates the response peaks at the higher sub-resonant speeds of $\left(v_{r} / 3, v_{r} / 4\right)$ 
due to the overestimated additional damping $(\Delta \zeta)$, as shown in Table 8 .

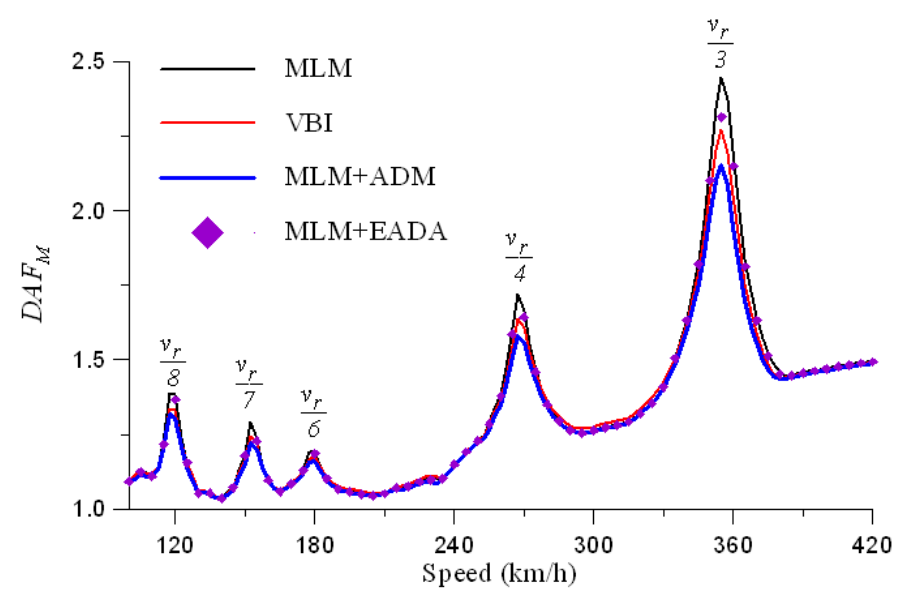

Fig. $13 D A F_{M}-v$ plot for the short bridge (S-12) subject to ICE2

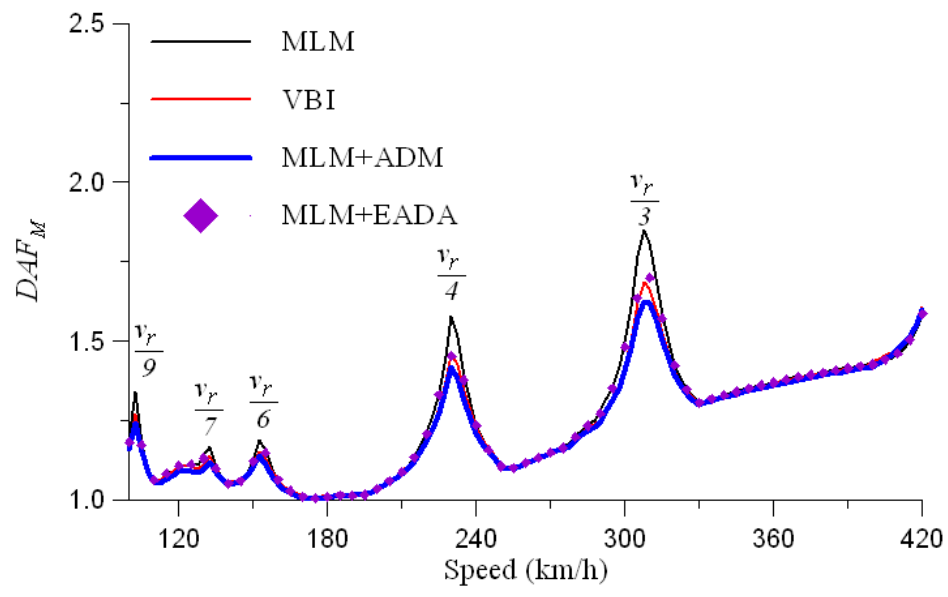

Fig. $14 D A F_{M}-v$ plot for the short bridge (S-14) subject to ICE2

Compared to the response curves in the $D A F_{M-V}$ plots depicted in Figs. 13 and 14 for the S-12 and S-14 bridges subject to the ICE-2 train, the sub-resonant peaks shown in Figs. 15 and 16 due to the ETR500Y train are almost identical for both of the MLM and the VBI models. This fact verifies that the interaction benefits would reduce or vanish on train-induced resonance of short bridges if low suspension stiffness is considered in a coach. 


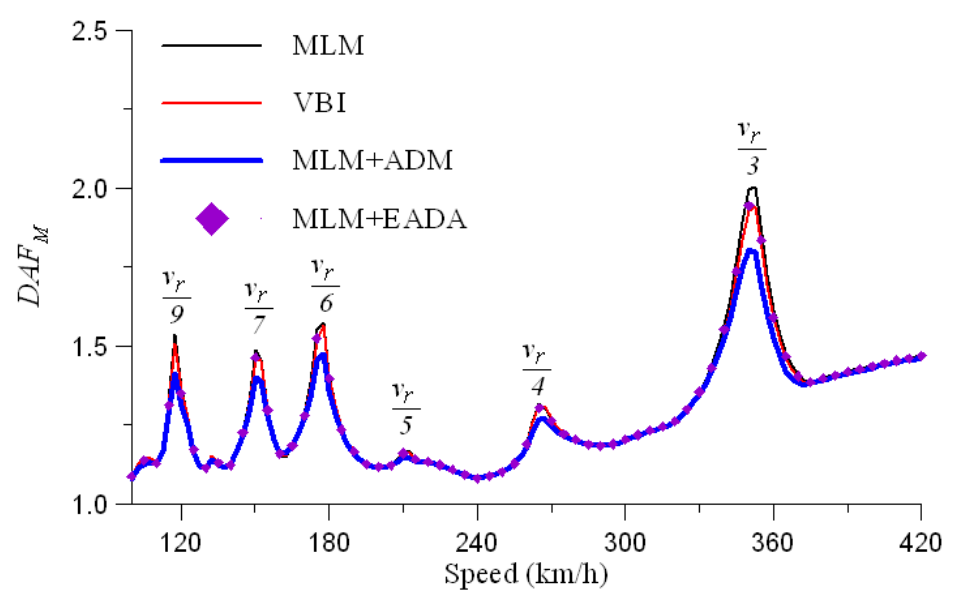

Fig. $15 D A F_{M}-v$ plot for the short bridge (S-12) subject to ETR500Y

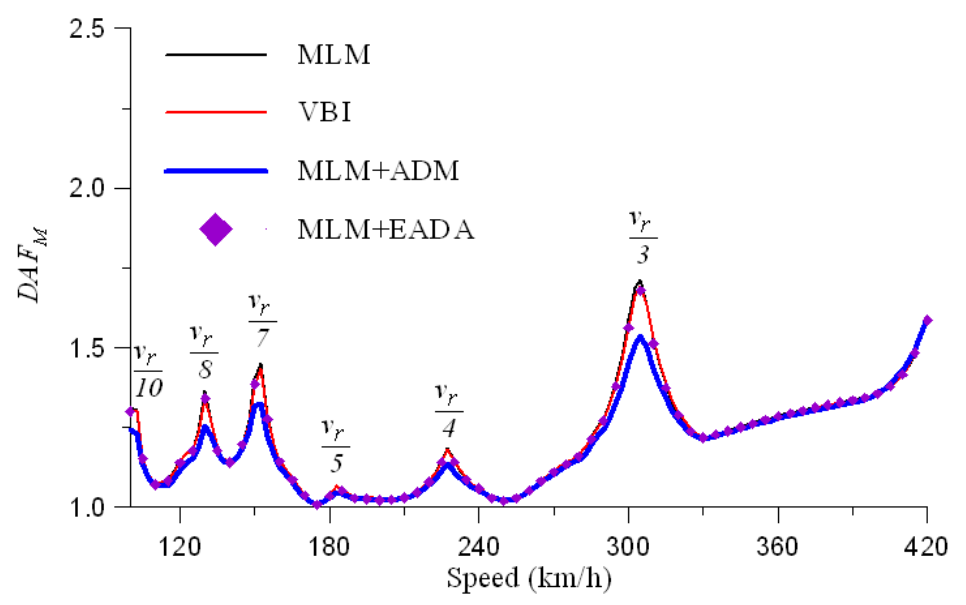

Fig. $16 D A F_{M}-v$ plot for the short bridge (S-14) subject to ETR00Y

From the parametric study conducted herein, it can be concluded that the proposed equivalent additional damping ratio analytical expression based on the non-dimensional parameters $\left(\mu_{1}, \xi_{v 1}, r_{1}\right)$ of the equivalent VBI system may serve as a reasonable estimation for the additional damping in short bridges, providing bridge engineers an alternative physical insight into the tuning effect of VBI dynamics on train-bridge systems. 


\section{Conclusions}

Due to the tuning effects of the suspension systems of trains traveling over railway bridges, the response of short bridges is reduced when the VBI effects are taken into account. To account for the VBI effects when the bridge response is analyzed using MLM, the ADM included in Eurocode 1 prescribes an additional damping that, in the case of short bridges, may lead to an excessive reduction of the maximum acceleration of the bridges under resonance conditions. For this reason, an alternative analytical approach based on an equivalent VBI model under resonant excitations is presented in this investigation with the aim of providing an accurate prediction of the interaction benefit. Here, the tuning model based on the first vertical vibration mode of the coach is considered for the equivalent VBI model. With the analytical model of the coach-bridge system, three non-dimensional key parameters $\left(\mu_{1}, \xi_{\mathrm{v} 1}, r_{1}\right)$ dominating the additional damping problem are subsequently identified. Depending on the values of these three ratios the interaction between the vehicles and the structure is more or less severe, and so is the level of additional damping that should be included in a model that just simulates the vehicles as constant loads.

The numerical investigations indicate that the proposed equivalent additional damping approach is a simple and feasible method and has enough accuracy for the MLM to account for VBI effect on short bridges when performing moving-load analyses. The following conclusions can be extracted from the numerical demonstrations and parametric studies conducted herein.

(1) As the coach suspension stiffness tends to a small value, the VBI benefits on the train induced resonant response of short bridges vanish. This is due to the fact that a low suspension stiffness may produce an isolating action and a reduction of the tuning effects of the vehicle masses on the beam vibration. 
(2) The sub-resonance peaks in the $a_{\max }-v$ plot (for maximum acceleration) of the beam will become denser with the reduction of the span lengths. Excessive vibrations could create negative conditions for ballast stabilization and train traffic safety for ballasted bridges.

(3) Since a short bridge presents much higher primary resonant speeds, a denser distribution of sub-resonance peaks in lower speed ranges should be expected on the $D A F_{M-V}$ plot for maximum bending moment. The influence of multiple sub-resonant conditions on short bridges should be taken into account in HSR design phases to evaluate the impact effect of a train moving on a short bridge at operation speeds.

(4) The present study can be regarded as a preliminary investigation of ADM on response analysis of short-span simply supported railway bridges. Following the present analytical approach, a further study can be carried out for more complicated types of short-span bridges, such as plate-frame bridges, using a refined bridge model.

\section{Acknowledgements}

The partial sponsorship from the Ministry of Science \& Technology of Taiwan via the grant numbers (MOST 106-2221-E-032-022, 107-2221-E-032-002-MY2), and the Taiwan-Czech joint project via the grant numbers (MOST 106-2923-E-032-007-MY3, GA CR 17-26353J) in conducting the research presented herein is gratefully acknowledged.

\section{References}

1. UIC Code 776-2 R, Design requirements for rail-bridges based on interaction phenomena between train, track and bridge. Union Internationale Des Chemins de Fer; 2009. 
2. European Committee for Standardization EN 1991-2, Actions on structures -Part 2: Traffic loads on bridges, CEN; Brussels, 2003.

3. ERRI D-214 Committee, Ponts rails pour vitesses $>200 \mathrm{~km} / \mathrm{h}$ et $<350 \mathrm{~km} / \mathrm{h}$. Conditions de deformation dynamiques, Rapport interimaire. Utrecht: European Rail Research Institute (ERRI); 1997.

4. Klasztorny M, Langer J, Dynamic response of single-span beam bridges to a series of moving loads. Earthq Eng Struct Dyna 1990;(19):1107-24.

5. ERRI D214, Rail bridges for speeds $>200 \mathrm{~km} / \mathrm{h}$. Final report. Part A: Synthesis of the results of D214 research, European Rail Research Institute; 1999.

6. Calgaro, JA, Tschumi, M, Gulvanessian H, Designer's guide to Eurocode 1: actions on bridges: EN 1991-2, EN 1991-1-1, -1-3 to -1-7 and EN 1990 annex A2. London: Thomas Telford; 2010.

7. Museros P, Romero ML, Poy A, Alarcon E, Advances in the analysis of short span railway bridges for high-speed lines. Comp \& Struct 2002;(80): 2121-32.

8. Doménech A, Museros P, Martínez-Rodrigo MD, Influence of the vehicle model on the prediction of the maximum bending response of simply-supported bridges under high-speed railway traffic. Eng Struct 2014;(72):123-39.

9. Yang YB, Yau JD, Resonance of high-speed trains moving over a series of simple or continuous beams with non-ballasted tracks. Eng Struct 2017;143:295-305.

10. Timoshenko S, Vibration problems in engineering. Primera edición: D. Van Nostrand Company Inc.; 1928.

11. Elishakoff I, Kaplunov J, Nolde E, Celebrating the centenary of Timoshenko's study of effects of shear deformation and rotary inertia. Appl Mech Rev 2015;(67):060802.

12. Yang YB, Yau JD, Vertical and pitching resonance of train cars moving over a series of simple beams. J Sound Vibr 2105;337:135-49. 
13. Yang YB, Yau JD, Hsu LC, Vibration of simple beams due to trains moving at high speeds. Eng Struct 1997;19:936-44.

14. Museros P, Alarcón E, Influence of the second bending mode on the response of high-speed bridges at resonance. ASCE J Struct Eng 2005;131: 404-15.

15. Yang YB, Yau JD, Vertical accelerations of simple beams due to successive loads traveling at resonant speeds. J Sound Vibr 2006;289:210-28.

16. Doménech A, Museros P, Influence of the vehicle model on the response of high-speed railway bridges at resonance. Analysis of the additional damping method prescribed by Eurocode 1. In: Proceedings of the 8th international conference on structural dynamics (EURODYN 2011) 2011;273-80.

17. Doménech A, Museros P, Nasarre J, Castillo-Linares A, Behavior of simply supported high-speed railway bridges at resonance: analysis of the influence of the vehicle model and simplified methods for dynamic analyses. In: Proceedings of the 25 th international conference on noise and vibration engineering (ISMA2012) 2012;1057-1072.

18. K. Liu, G. De Roeck, G. Lomabaert, The effect of dynamic train-bridge interaction on the bridge response during a train passage, J. Sound Vibr 2009;325:240-251.

19. Newmark NM, A method of computation for structural dynamics. ASCE J Eng Mech Div 1959;85: 67-94.

20. Museros P, Moliner E, Martínez-Rodrigo M, Free vibrations of simply supported beam bridges under moving loads: maximum resonance, cancellation and resonant vertical acceleration. J Sound Vibr 2013;332:326-45.

21. Xia H, Li HL, Guo WW, De Roeck G, Vibration resonance and cancellation of simply supported bridges under moving train loads. ASCE J Eng Mech $2013 ; 04014015$.

22. Yang YB, Yau JD, Wu YS, Vehicle-bridge interaction dynamics with application 
to high-speed railways, World Scientific Publishing; Singapore 2004.

\section{Appendix:}

With reference to Fig. 3, the following symbols are used: $D=$ length of the car body, $d=$ axle interval between the two bogies, $b=$ bogie length, $\left(M_{v}, I_{v}\right)=$ lumped mass and pitching inertia of the car body, $\left(m_{b}, I_{b}\right)=$ lumped mass and pitching inertia of each bogie, $m_{w}=$ mass of each wheelset, $\left(k_{p}, C_{p}\right)=$ primary suspension stiffness and damping in each bogie, $\left(k_{s}, c_{s}\right)=$ secondary suspension stiffness and damping to support the car body, $\left(u_{v}, \theta_{v}\right)=$ vertical displacement and pitching rotation at midpoint of the car body, and $\left(u_{b j}, \theta_{b j}\right)=$ vertical displacement and pitching rotation at midpoint of the $j$-th bogie.

$\mathbf{M}_{\mathbf{c}}=\left[\begin{array}{cccccc}M_{v} & & & & & \\ & I_{v} & & & & \\ & & m_{b} & & & \\ & & & I_{b} & & \\ & & & & m_{b} & \\ & & & & & I_{b}\end{array}\right]$

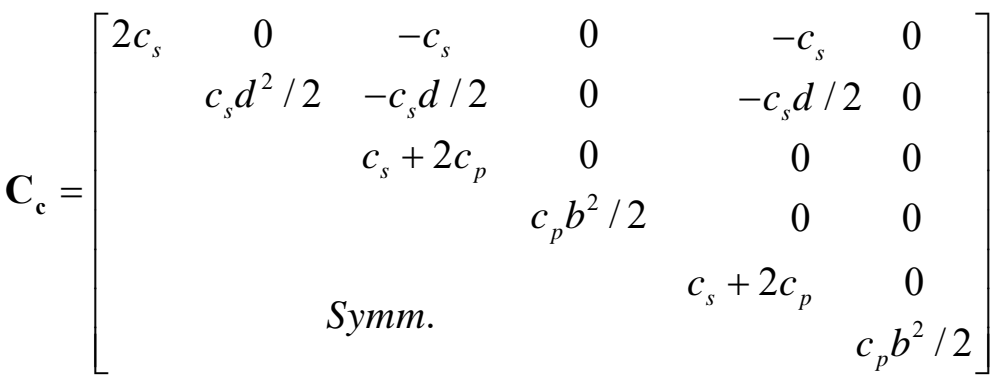

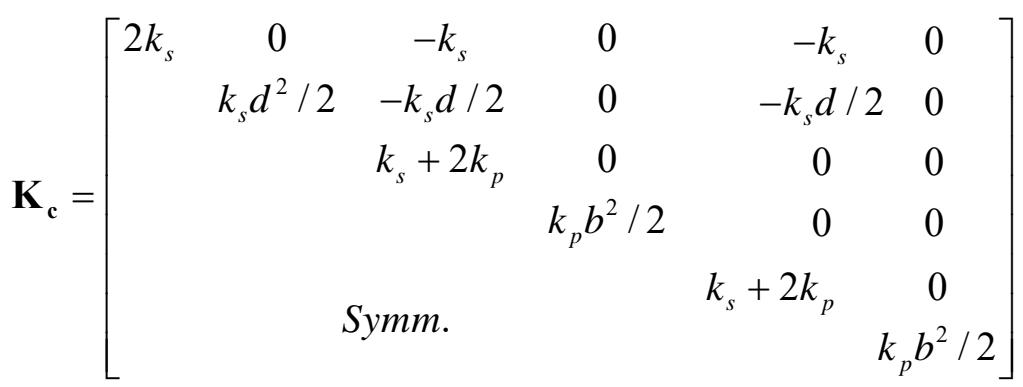




$$
\mathbf{u}_{\mathbf{c}}=\left\{\begin{array}{c}
u_{v} \\
\theta_{v} \\
u_{b 1} \\
\theta_{b 1} \\
u_{b 2} \\
\theta_{b 2}
\end{array}\right\}, \mathbf{f}_{\mathbf{c}}=\left\{\begin{array}{c}
0 \\
0 \\
k_{p}\left(u\left(x_{11}, t\right)+u\left(x_{21}, t\right)\right) \\
k_{p} b\left(u\left(x_{11}, t\right)-u\left(x_{21}, t\right)\right) / 2 \\
k_{p}\left(u\left(x_{12}, t\right)+u\left(x_{22}, t\right)\right) \\
k_{p} b\left(u\left(x_{12}, t\right)-u\left(x_{22}, t\right)\right) / 2
\end{array}\right\}+\left\{\begin{array}{c}
0 \\
0 \\
c_{p} b\left(\dot{u}\left(x_{11}, t\right)-\dot{u}\left(x_{21}, t\right)\right) / 2 \\
c_{p}\left(\dot{u}\left(x_{12}, t\right)+\dot{u}\left(x_{22}, t\right)\right) \\
c_{p} b\left(\dot{u}\left(x_{12}, t\right)-\dot{u}\left(x_{22}, t\right)\right) / 2
\end{array}\right\}
$$

Here $x_{i j}$ stands for the position of the $i$-th wheel-set in the $j$-th bogie on the beam. 